\title{
CS-free food contingencies and subsequent acquisition of conditioned suppression: No transfer effect*
}

\author{
DONALD E. JACKSON \\ Eastern Michigan University, Ypsilanti, Michigan 48197
}

\begin{abstract}
Following VI training for food, 32 rats received 12 CSs (levers retracted) over 2 days during which three of four groups received free food. CS-free food contingencies were positive, negative, or absent; the fourth group received CSs alone. Subsequent CER acquisition (3 days) on the baseline revealed only a significant trials effect. These findings question the generality of the transfer-of-control phenomenon.
\end{abstract}

Recent investigators have reported an effect of response-independent CS-free food contingencies on subsequent avoidance behavior. Grossen, Kostansek, and Bolles (1969) trained rats on a free operant avoidance task (shuttling). Two of their groups were then given free food either following a tone (CS+) or during nontone periods only (CS-). When later tested in avoidance extinction, tone trials produced slower avoidance responding for CS+ Ss and faster responding for CS - Ss compared to toneless trials. Similar findings were reported by Bull (1970). More recently, Overmier, Bull, and Pack (1971) found that previously conditioned auditory CSs would affect subsequently acquired avoidance shuttling in dogs. In this study, CS+ had merely signaled the availability of response-contingent food; CS- was used as the cue for pedal-pressing avoidance in different Ss. The auditory CS suppressed shuttle avoidance for CS+ Ss and facilitated shuttling for CS- Ss. Thus, suppression of avoidance in the presence of CS+ has been found regardless of whether the avoidance training came before (Grossen et al, 1969) or after (Overmier et al, 1971) the CS-food relationship was established.

On balance, the results of these transfer-of-control studies support a centrally mediated, response-independent hypothesis. The purpose of the present study was to test the generality of the transfer-of-control phenomenon using rats in a conditioned suppression paradigm. Response-independent food was presented in different contingent relationships with the CS which was subsequently paired with shock during acquisition of conditioned suppression (CER). Transfer of control would be demonstrated if groups differentially acquired CER as a function of the CS-free food contingency.

\section{METHOD}

\section{Subjects}

Ss were 32 naive male albino rats (Sprague-Dawley) obtained from Spartan Research Animals, Haslett, Michigan. Weights

\footnotetext{
*These data were presented at the 1973 Meeting of the
} American Psychological Association, Montreal. ranged from $259-296 \mathrm{~g}$ (mean $=272 \mathrm{~g}$ ) at the beginning of the experimentation. Ss were individually housed under constant illumination with unlimited access to water. Ss were weighed daily and maintained at $85 \%$ of their free-feeding weights through limited feeding of Purina Rat Chow 30 min after each session.

\section{Apparatus}

All testing was done in eight identical Lehigh Valley dual-bar chambers (right lever permanently retracted). Each chamber $(30.5 \times 24 \times 27 \mathrm{~cm})$ was housed in a blower-ventilated, sound-attenuating enclosure and was located in one of eight separate rooms.

Each unit was equipped with a speaker mounted on the enclosure wall $30 \mathrm{~cm}$ from the lever, shocker (LVE 113-04), and solid state control modules. Reinforcement (45-mg Noyes pellets) availability and CS presentations were controlled from a central source in an adjacent room where printout counters were located.

The CS was a 2-min white noise produced by two Grason-Stadler noise generators (Model 901B). Each generator served four chambers. Noise level readings $(80 \pm 1 \mathrm{~dB}$ re $.0002 \mathrm{dynes} / \mathrm{cm}^{2}$ ) were taken at the response lever. The US was a $0.5-\mathrm{sec}, 1.5-\mathrm{mA}$ scrambled shock delivered through floor grids of $0.48-\mathrm{cm}$-diam stainless steel rods $1.90 \mathrm{~cm}$ apart.

\section{Procedure}

Preliminary Training. Ss self-trained to a criterion of 50 reinforcements (each response produced a pellet). Shaping was used where necessary. The following day, VI 1-min training began and consisted of nine 45-min daily sessions.

CS-Free Food Delivery. The next day Ss were randomly assigned to four treatment groups balanced for chamber and time of day effects. With response levers retracted, Ss received six 2-min CSs and free pellets as follows: (a) Group 1-0 received 10 pellets per CS, (b) Group 0-1 received 10 pellets during each of six selected non-CS periods, (c) Group 1-1 received pellets during both CS and non-CS periods (from 2-4 during each CS), and (d) Group 0-0 received CSs only. CS onsets occurred at 4, $10,18,25,34$, and $39 \mathrm{~min}$ into the $45-\mathrm{min}$ session. The free-food procedure was repeated the next day with CS onsets at $3,7,17,26,32$, and 38 min into the session. Thus, all Ss except those in Group 0-0 received 120 free pellets over the 2-day treatment period.

Baseline Recovery. Two operant recovery days followed with the original VI 1-min reinforcement schedule in effect for all Ss.

Acquisition of Conditioned Suppression. CER acquisition began the next day and consisted of four daily CS-US pairings for 3 days (45-min sessions). Response levers were available and the shock US occurred as the 2-min CS terminated. ITIs were irregular within and between days and averaged approximately $10 \mathrm{~min}$ 


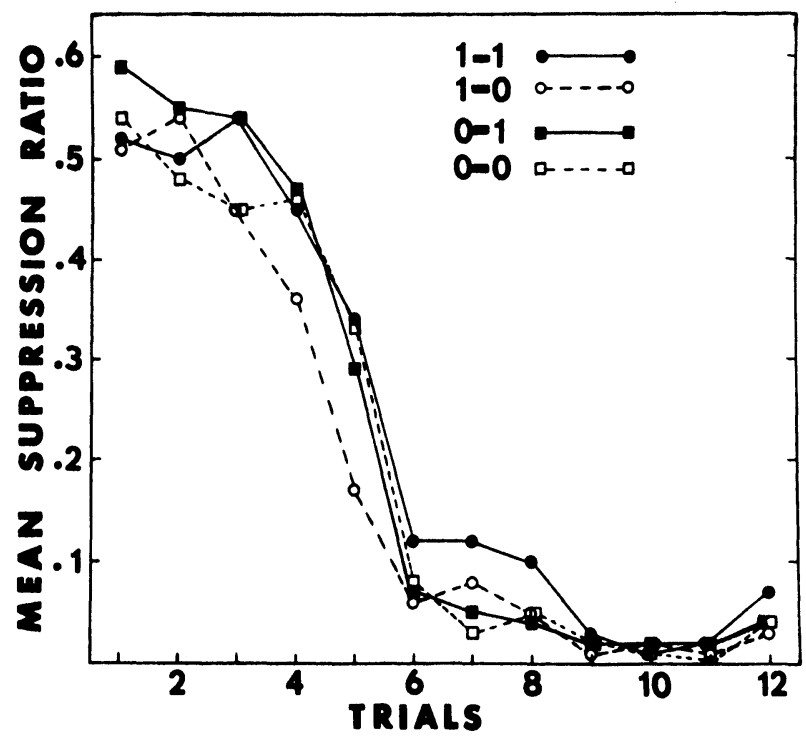

Fig. 1. Mean suppression ratios during CER acquisition.

CER Extinction. The next day Ss were given six extinction trials (CSs only) with an average ITI of $6 \mathrm{~min}$.

Index of Suppression. Response suppression was indexed by the ratio, $\mathrm{B} /(\mathrm{A}+\mathrm{B})$, where $\mathrm{A}$ and $\mathrm{B}$ refer to number of responses emitted during 2-min pre-CSs and CSs, respectively. In addition, a measure of generalized suppression was obtained by comparing pre-CS (A) responses during CER acquisition with a response index based on the last 3 days of VI training (prior to free food).

\section{RESULTS}

Three repeated-measures analyses of variance were performed: (a) on suppression ratios obtained during CER acquisition, (b) on generalized suppression ratios obtained during CER acquisition, and (c) on suppression ratios obtained during CER extinction. The results of each analysis were basically the same: no significant treatment or Treatment by Trials effects (Fs ranged from 0.4-1.2), but highly significant trials effects. The trials Fs were 145.7 (CER acquisition), 5.7 (generalized suppression), and 32.7 (CER extinction). Figure 1 shows the suppression ratios obtained during CER acquisition.

\section{DISCUSSION}

These results are not in accord with those studies which have demonstrated a transfer-of-control effect in an avoidance situation. However, those studies have tested for transfer of control during conditions of avoidance extinction. The avoidance transfer-of-control paradigm analogous to the present study would involve using the same CS in active signaled avoidance training that had been used in the Pavlovian phase (CS-free food). Failure to find a transfer effect might indicate that shock is too disruptive for the transfer effect to be demonstrated in its presence. On the other hand, additional CS-free food sessions prior to CER acquisition may have led to different results in the present study. However, the present results are in basic agreement with Poppen (1970) who found that CS-contingent free food pellets given during CER extinction did not reduce the number of trials to a no-suppression criterion compared to regular CER extinction control Ss. Poppen's study represents the other side of the transfer question by testing for free food effects on extinction of CER.

Failure to find a transfer effect suggests that the generality of the phenomenon is restricted. Its limitations need to be specified both in terms of the aversive paradigms in which the effect can be demonstrated and in terms of the amount of Pavlovian conditioning (CS-free food pairings) required.

\section{REFERENCES}

Bull, J. A., III. An interaction between appetitive Pavlovian CSs and avoidance responding. Learning \& Motivation, 1970, 1 , 18-26.

Grossen, N. E., Kostansek, D. J., \& Bolles, R. C. Effect of appetitive discriminative stimuli on avoidance behavior. Journal of Experimental Psychology, 1969, 81, 340-343.

Overmier, J. B., Bull, J. A., \& Pack, K. On instrumental response interaction as explaining the influences of Pavlovian $\mathrm{CS}^{+}$ upon avoidance behavior. Learning \& Motivation, 1971, 2, 103-112.

Poppen, R. Counterconditioning of conditioned suppression in rats. Psychological Reports, 1970, 27, 659-671.

(Received for publication July 1, 1974.) 